\title{
Technology Corner: Virtual Crime Scene Reconstruction: The Basics of 3D Modeling
}

Nick V. Flor

University of New Mexico

Follow this and additional works at: https://commons.erau.edu/jdfs|

Part of the Computer Engineering Commons, Computer Law Commons, Electrical and Computer Engineering Commons, Forensic Science and Technology Commons, and the Information Security Commons

\section{Recommended Citation}

Flor, Nick V. (2011) "Technology Corner: Virtual Crime Scene Reconstruction: The Basics of 3D Modeling," Journal of Digital Forensics, Security and Law. Vol. 6 : No. 4 , Article 6.

DOI: https://doi.org/10.15394/jdfsl.2011.1108

Available at: https://commons.erau.edu/jdfsl/vol6/iss4/6

This Article is brought to you for free and open access by the Journals at Scholarly Commons. It has been accepted for inclusion in Journal of Digital Forensics, Security and Law by an authorized administrator of Scholarly Commons. For more information, please contact commons@erau.edu.

(c)ADFSL 


\title{
Technology Corner
}

\section{Virtual Crime Scene Reconstruction: The Basics of 3D Modeling}

\author{
Nick V. Flor \\ Information Systems, Film, \& Digital Media \\ Anderson School of Management \\ University of New Mexico \\ nickflor@unm.edu
}

\begin{abstract}
Digital crime scenes take place in the context of physical crime scenes. Virtual crime scene reconstruction is an activity where investigators create a 3dimensional (3-D) model of an actual crime scene for the purpose of determining the events that lead to the crime. While virtual crime scene reconstruction is currently used for analyzing physical scenes, it can also help investigators visualize and explore ways digital media could have been used to perpetrate a crime. In this technology corner we explore one of the technologies underlying virtual crime scene reconstruction: 3-D modeling.
\end{abstract}

\section{VIRTUAL CRIME SCENE RECONSTRUCTION}

Virtual Crime Scene Reconstruction is an activity where an investigator or an investigative team creates a 3-dimensional (3-D) model of an actual crime scene for the purpose of determining the events that lead to the crime. Crime scene reconstruction, in general, is the fifth and final phase of a crime scene investigation (Carrier \& Spafford, 2003), and the stages of reconstruction include conjecture, hypothesis formulation, testing, and theory formulation (Miller, 2009). Virtual crime scene reconstruction can assist with all these stages.

While the focus of this journal is digital crime scenes, such crimes take place in the context of physical crime scenes. For example, the National Institute of Justice describes digital media in the context of the following fourteen physical crime categories (National Institute of Justice, 2008): child abuse or exploitation; computer intrusion; counterfeiting; death investigation; domestic violence, threats, and extortion; e-mail threats, harassment, and stalking; gambling; identity theft; narcotics; online or economic fraud; prostitution; software piracy; telecommunications fraud; and terrorism (homeland security).

For these crime categories and others, virtual reconstruction can help investigators visualize and explore ways digital media could have been used to perpetrate a crime. 


\section{WAYS TO RECONSTRUCT A CRIME SCENE IN 3-D}

There are many different ways of reconstructing a crime scene. Automated techniques combine software with laser scanning (LIDAR), with multiple photographs, with video sequences, and with stereoscopic photographs (Se \& Jasiobedzki, 2005). However, the technique I will discuss is manual modelingby hand - as it is the least expensive, the most flexible, and can be used to augment the automated techniques. Moreover, skill in manual modeling is useful beyond crime scene constructions, as it can be used to create animated visualizations of digital crime scenes that are difficult to describe. According to cognitive researchers, visualizations and other forms of external representations can significantly assist problem solving processes (Larkin \& Simon, 1987).

\section{CHOOSING MODELING SOFTWARE-FREELY-AVAILABLE PACKAGES}

Popular commercial modeling software packages include Maya and 3ds-Max, both by Autodesk Corporation, while the most popular open-source modeling software package is Blender. Both Maya and 3ds Max are expensive software packages, as they are used to create models for both Hollywood movies and videogames. However, Autodesk makes them available freely, with full functionality; the only restriction is that anything created by the software is for academic-use only. Instructors and students can download these packages from students.autodesk.com, after registering with an e-mail address from a recognized academic institution. Blender can be downloaded from www.blender.org.

Most all 3-D modeling software packages are sufficient for 3-d crime scene construction. The decision of what package to use depends largely on the computers available in one's organization or lab - as not all modeling software will run on all operating systems. In particular, both Maya and Blender run on the Windows, the Mac, and the Linux operating systems. 3ds Max runs only on the Windows operating system.

\section{THE BASICS OF 3-D MODELING}

There are two basic ways of modeling: polygons modeling and curve-based modeling. We will focus on polygon modeling, which is the most common type of modeling.

\subsection{Basic Primitives}

With polygon modeling, the person modeling (hereafter, the "modeler") starts by adding a basic primitive to the workspace of the software. In most modeling tools, the basic primitives are spheres, cubes, cylinders, cones, and flat planes. The virtual crime scene modeler uses both the crime scene documentation and these basic primitives to start modeling a scene.

Suppose, for example, the crime scene took place in a living room, and that measurements made during the crime scene documentation phase, indicated that 
the room was $15 \times 15$ feet in area, with 9-foot tall walls. A crime scene modeler would start off by creating a cube was $15 \times 15 \times 9$ units in the modeling tool's workspace (see Figure 1).

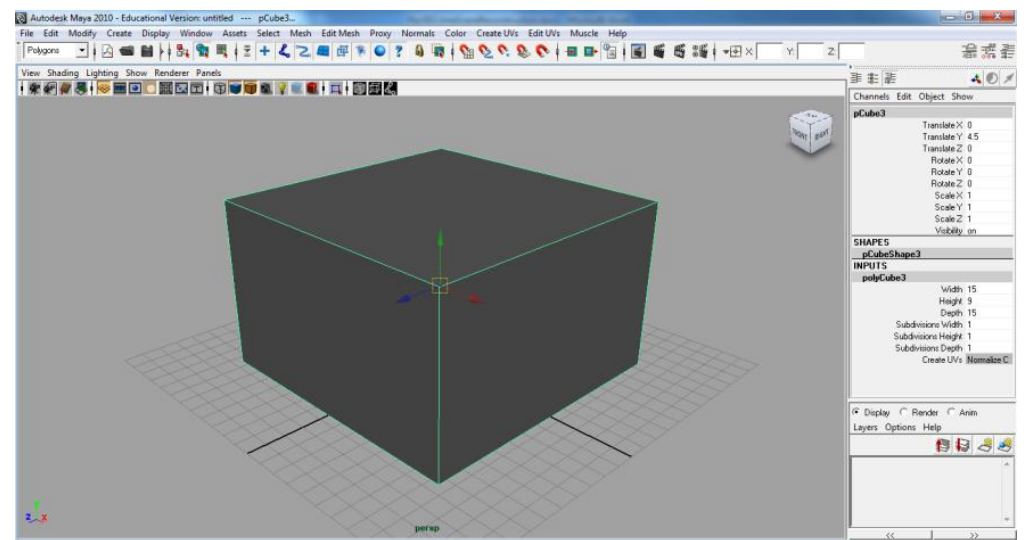

Figure 1. A basic cube primitive representing a living room that is 15 feet wide, by 15 -feet deep by 9 feet tall.

Within the room there would be a variety of furniture and electronics. These everyday objects would be modeled next, based again on measurements from the crime scene documentation.

\subsection{Creating Everyday Objects from Basic Primitives}

Aside from rooms and certain appliances like toasters, most objects are not cubes. Nevertheless, the modeler still starts with a cube and then adds both edge loops and extrusions to mold the cube into the object. An edge loop is a line that goes completely around an object, while an extrusion is a protrusion off one or more faces of an object.

To see how edge loops and extrusions work, suppose the living room had a 4legged coffee table in the center. To model the coffee table, the modeler first starts by modeling the table top as a flat cube (see Figure 2).

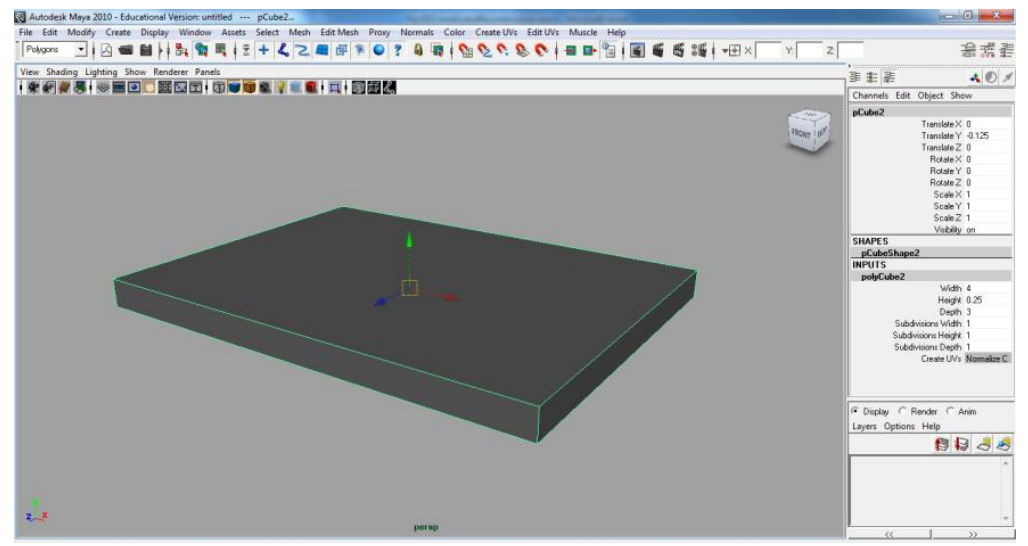


Figure 2. Coffee table top as a flat cube.

To complete the coffee table, the modeler add four legs as extrusion off of the table top. The problem, however, is that extrusions operate on faces, and there are no faces on the coffee table top that are suitable for extruding as legs. By adding edge loops, a modeler creates faces on the table top that can be extruded to depict legs. Figure 3 depicts the coffee table with several edge loops added.

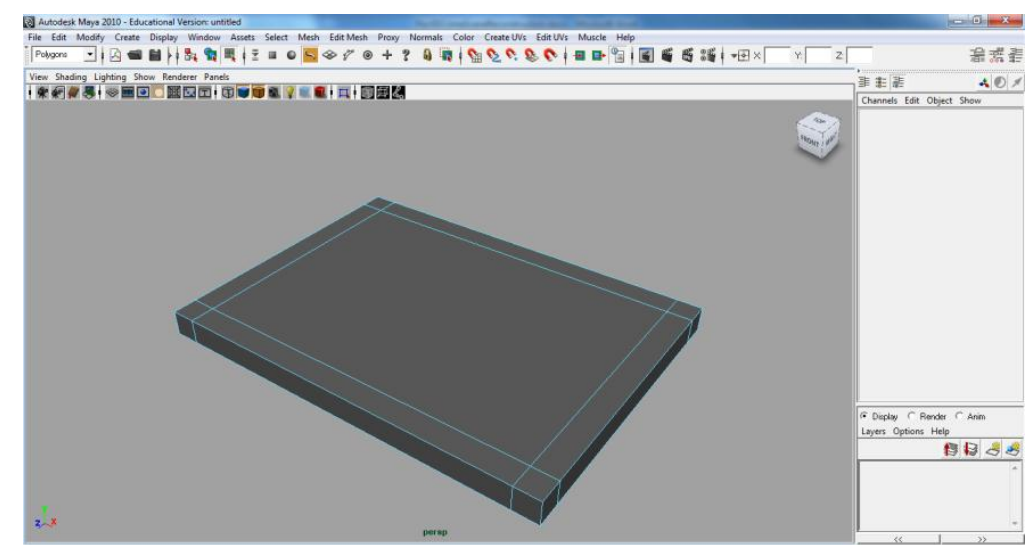

Figure 3. Edge loops added to the table top (compare with Figure 2) so that legs can be extruded.

Note that there are now four faces in the corners of the table top that a modeler can use for extrusions, but this is the table top and the legs are located on the bottom. Fortunately, since edge loops go all the way around an object, there are also four faces in the corners of the table bottom (see Figure 4, left), which a modeler can use to extrude legs. Figure 4 (right) shows the results of using the extrude operation to create legs for the coffee table. 


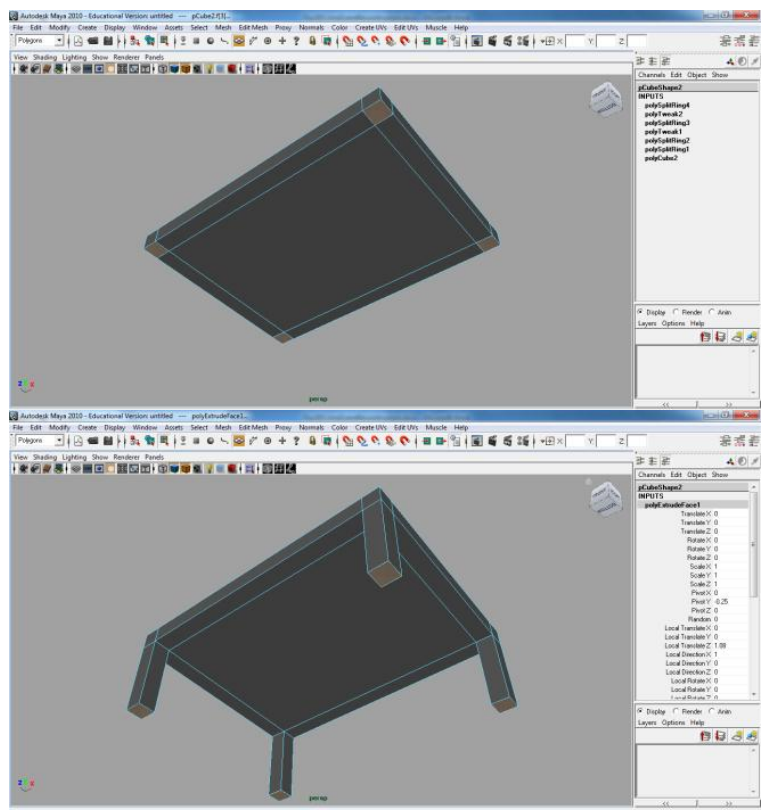

Figure 4. Coffee table bottom prior to extrusions (left). Extrusions used to create legs (right).

With the coffee table created, it can be imported into the center of the room (see Figure 5). Modelers, generally, create distinct objects in separate files and then import these objects into a main scene file. This allows modelers to reuse objects and also to create more complex objects based on existing models.

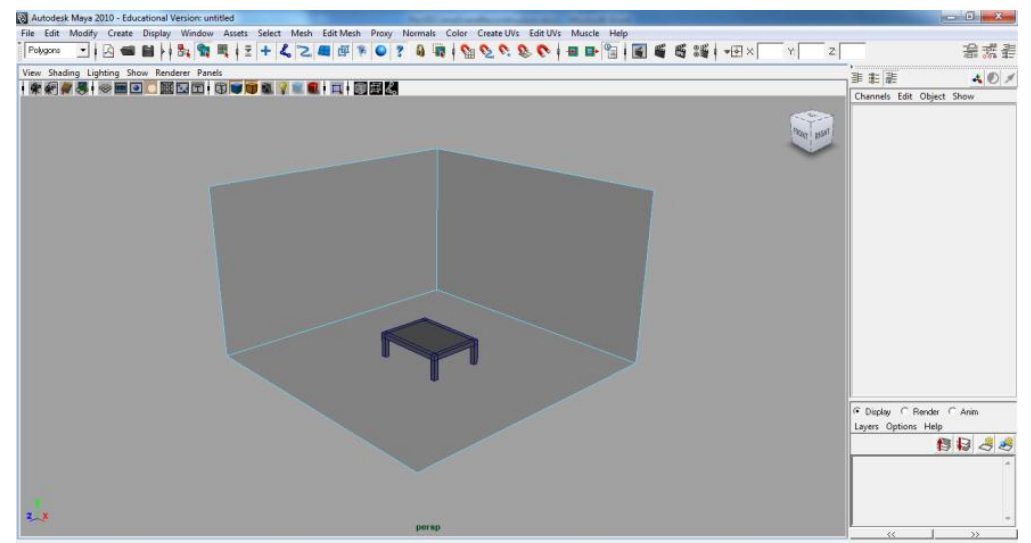

Figure 5. Coffee table placed into the room (walls hidden to see inside the room).

\subsection{Reusing Complex Models to Create New Objects}

Instead of always starting with basic primitives like cubes to create objects, one can start with complex models like the coffee table, and then add edge loops and extrusions - just like adding them to a basic primitive - to create even more 
complex objects. Figure 6 depicts both a couch (middle image) and a bed (right image) that were created by using the coffee table as a starting point (left image) and adding extrusions; no extra edge loops were added to the coffee table. Creating the bed also made use of a move-vertices operation, where the vertices for the coffee table's front legs were selected and pulled out to stretch the coffee table into a bed.

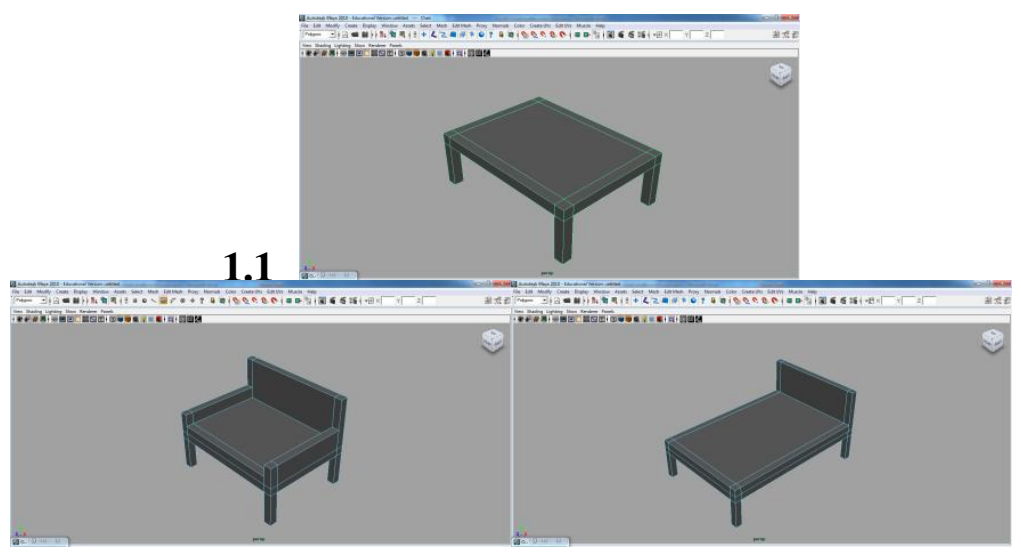

Figure 6. A more complex object, the coffee table (right) used as the starting point for a couch (middle) and a bed (left)

\subsection{Creating the Scene}

With the room and all the objects in the room modeled according to the measurements in the crime scene documentation, they are imported into a single file that represents the virtual crime scene. Figure 7 depicts such a scene with several sofas, a coffee table and an entertainment center.

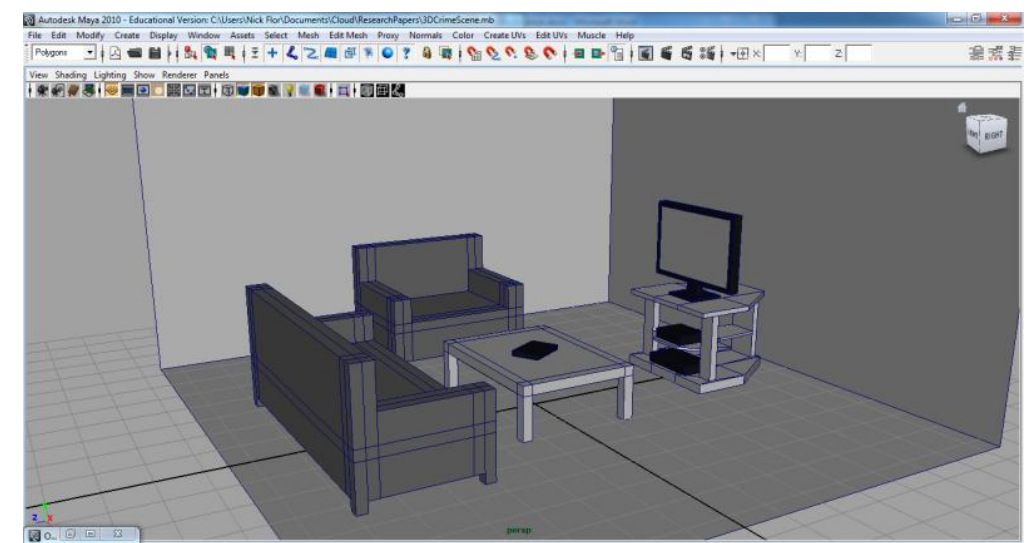

Figure 7. An entire room made with just cubes, edge loops, extrusions, and vertex movement. 


\section{SUMMARY \& CONCLUSION}

I have described the fundamentals of 3-D virtual crime scene reconstruction using manual modeling. As was demonstrated, one could represent a crime scene with just a cube, edge loops, extrusions, and vertex movement, along with the measurements from the crime scene documentation. 3-D modeling is only the first step in virtual crime scene reconstruction. Other steps include:

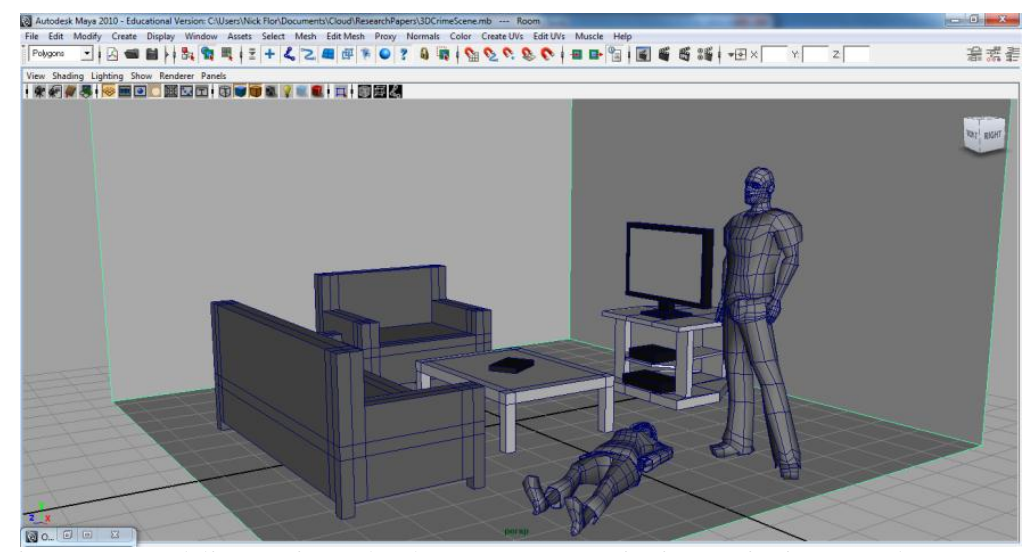

Figure 8. Adding Virtual Characters Depicting Victims and Suspects

1. Modeling characters that depict suspects, victims, and witnesses in the crime scene (see Figure 8). It turns out that to model a characters, one still uses cubes, edge loops, extrusions, and vertex movements, but the process also requires full-body and full-face reference pictures, in both front and side views.

2. Texture mapping the objects and characters. Modeled objects and characters are a dull gray. To create realistic looking characters with color, one must wrap texture maps over the models. Texture maps are pictures that are "pasted" over the models. These pictures can be taken from the photographs in the crime scene documentation.

3. Animating characters and moving objects. In a virtual crime scene there is activity and movement. Moving characters and moving objects requires adding a "rig" - a kind of skeleton with joints that can be rotated to make the model appear to move.

4. Finally, there is scripting, or writing code, to trigger movements and animations.

In future Technical Corners, we plan to explore these and other more digital forensics oriented topics. I hope the past couple of Technical Corners have given 
you, the reader, a sense of the breadth of topics possible in this section of the journal. We have covered packet sniffing, e-mail headers, brute-force password generation, and virtual crime scene reconstruction. I look forward to future submissions from readers that describe technologies and procedures used either in the classroom or in the field.

\section{REFERENCES}

Carrier, B., \& Spafford, E. (2003). Getting Physical with the Digital Investigation Process. International Journal of Digital Evidence , 2(2), 1-20.

Miller, M. T. (2009). Crime Scene Investigation. In S. H. James, \& J. J. Nordby, Forensic Science: An Introduction to Scientific and Investigative Techniques (pp. 167-192). Boca Raton, FL: CRC Press.

National Institute of Justice. (2008, April 9). Electronic Crime Scene Investigation: A Guide for First Responders, Second Edition. Retrieved January 10, 2012, from U.S. Department of Justice: http://www.nij.gov/publications/ecrime-guide-219941/

Se, S., \& Jasiobedzki, P. (2005). Instant Scene Modeler for Crime Scene Reconstruction. Proceedings of the 2005 IEEE Computer Society Conference on Computer Vision and Pattern Recognition.

Why a Diagram is (Sometimes) Worth Ten Thousand Words. (1987). Cognitive Science, 11, 65-99. 